\title{
Novel Refrigerator Development
}

J. Ullom, M.F. Cunningham, O.B. Drury, M. Frank, S. Friedrich, S.E. Labov, P.A. Fisher and P. Gifford

This article was submitted to $8^{\text {th }}$ International Workshop on Low Temperature Detectors, Dalfsen, The Netherlands, August 15-20, 1999

\section{August 30, 1999}

U.S. Department of Energy

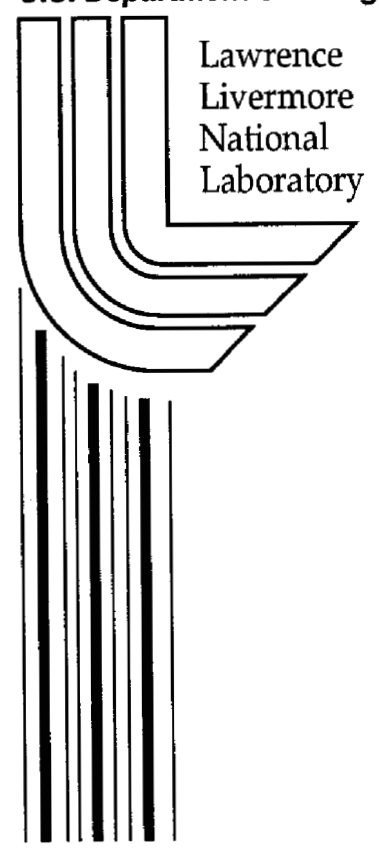




\section{DISCLAIMER}

This document was prepared as an account of work sponsored by an agency of the United States Government. Neither the United States Government nor the University of California nor any of their employees, makes any warranty, express or implied, or assumes any legal liability or responsibility for the accuracy, completeness, or usefulness of any information, apparatus, product, or process disclosed, or represents that its use would not infringe privately owned rights. Reference herein to any specific commercial product, process, or service by trade name, trademark, manufacturer, or otherwise, does not necessarily constitute or imply its endorsement, recommendation, or favoring by the United States Government or the University of California. The views and opinions of authors expressed herein do not necessarily state or reflect those of the United States Government or the University of California, and shall not be used for advertising or product endorsement purposes.

This is a preprint of a paper intended for publication in a journal or proceedings. Since changes may be made before publication, this preprint is made available with the understanding that it will not be cited or reproduced without the permission of the author.

This work was performed under the auspices of the United States Department of Energy by the University of California, Lawrence Livermore National Laboratory under contract No. W-7405-Eng-48.

This report has been reproduced directly from the best available copy.

Available electronically at http://www.doc.gov/bridge

Available for a processing fee to U.S. Department of Energy

And its contractors in paper from

U.S. Department of Energy

Office of Scientific and Technical Information

P.O. Box 62

Oak Ridge, TN 37831-0062

Telephone: (865) 576-8401

Facsimile: (865) 576-5728

E-mail: reports@adonis.osti.gov

Available for the sale to the public from

U.S. Department of Commerce

National Technical Information Service

5285 Port Royal Road

Springfield, VA 22161

Telephone: (800) 553-6847

Facsimile: (703) 605-6900

E-mail: orders@ntis.fedworld.gov

Online ordering: http://www.ntis.gov/ordering.htm

Or

Lawrence Livermore National Laboratory

Technical Information Department's Digital Library

http://www.llnl.gov/tid/Library.html 


\title{
NOVEL REFRIGERATOR DEVELOPMENT
}

\author{
J.N. Ullomª , M.F. Cunningham ${ }^{2}$, O.B. Drury ${ }^{2}$, M. Frank ${ }^{2}$, S. Friedrich ${ }^{2}$, S.E. Labov ${ }^{a}$ \\ P.A. Fisher ${ }^{b}$, P. Gifford ${ }^{c}$ \\ at Lawrence Livermore National Laboratory, Livermore CA, 94550, USA \\ ${ }^{b}$ Analog Devices Corp., MS426, Wilmington MA, 01887, USA \\ ${ }^{\mathrm{c} C}$ Cryomech Inc., 113 Falso Drive, Syracuse NY, 13211, USA
}

\begin{abstract}
In part $\mathrm{I}$, we describe how the creation of quasiparticles by current flow through a normal-insulatorsuperconductor (NIS) junction degrades the cooling performance of the junction. Degradation occurs due to the absorption of recombination phonons in the normal electrode and due to a reduction in the cooling power. In part II, we describe how vibrations from a pulse tube mechanical cooler affect X-ray measurements performed with a superconducting tunnel junction.
\end{abstract}

\section{I-NIS COOLING}

Since the first demonstration of electron cooling by NIS tunnel junctions [1], there has been interest in the development of on-chip refrigerators having base temperatures near $100 \mathrm{mK}$ when operated from bath temperatures of several hundred $\mathrm{mK} .[2,3]$ Here, we show how heating of the electron system in the superconducting electrode of a NIS junction can degrade refrigerator performance.

Current flow through a NIS tunnel junction heats the electron system in the superconducting electrode through the creation of quasiparticles. This heating degrades the cooling performance of the junction in two ways. First, quasiparticle recombination produces phonons which can travel through the tunnel barrier and heat the normal electrode. Second, the power removed from the normal electrode decreases as the quasiparticle density in the superconductor increases. Electrons which tunnel from below the Fermi level of the normal metal and fill hole-like excitations in the superconductor are especially detrimental to the cooling power. The two degradation mechanisms are described quantitatively below.

If the quasiparticle density in the superconducting electrode is $n_{x}+n_{t h}$ where $n_{t h}$ is the thermal density, then the recombination rate is $\Gamma_{R}\left(n_{x}+n_{t h}\right)^{2} V_{S}$ quasiparticles per second where $\Gamma_{R}$ is the recombination rate per unit density and $V_{S}$ is the volume. The power load on the normal electrode due to phonons is then given by $\Gamma_{R}\left(n_{x}^{2}+2 n_{x} n_{t h}\right) V_{S} \Delta p_{p-e}$ where $\Delta$ is the energy gap and $p_{p-e}$ is the probability a phonon excites an electron in the normal electrode.

The power deposited in the superconducting electrode of a NIS junction is given by

$$
\begin{array}{r}
P_{S}=\frac{1}{e^{2} R_{N}} \int_{\Delta}^{\infty} E N(E)\left[f_{N}\left(E+e V_{b}\right)+f_{N}\left(E-e V_{b}\right)\right. \\
\left.-2 f_{S}(E)\right] d E
\end{array}
$$

where $R_{N}$ is the resistance, $N(E)$ is the energydependence of the superconducting density of states, 
$V_{b}$ is the bias voltage, and $f_{N}\left(f_{S}\right)$ is the Fermi function in the normal (superconducting) electrode. We assume that the electrons in the normal metal (superconductor) have an effective temperature $T_{N}$ $\left(T_{S}\right)$. The cooling power in the normal electrode, $P_{N}$, is $P_{S}-I V_{b}$. In Fig. 1, we plot the normalized cooling power as a function of $T_{S} / T_{N}$ for $T_{N}$ equal to $0.1,0.2$, and $0.3 \mathrm{~K}$ and $\Delta=180 \mu \mathrm{eV}$. The cooling power decreases when $T_{S}$ exceeds $T_{N}$. The loss in cooling power due to heating in the superconductor is given by $P_{N}\left(T_{N}, T_{S}\right)-P_{N}\left(T_{N}, T_{S}=T_{b}\right)$ where $T_{b}$ is the bath temperature.

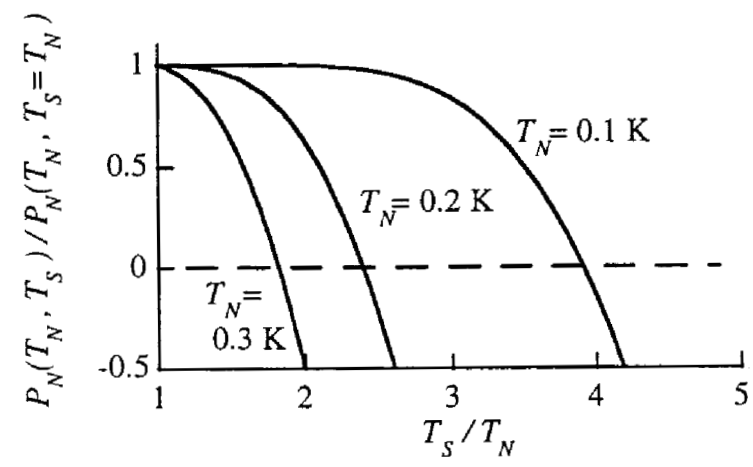

fig. 1 Cooling power $P_{N}$ with normal electrode temperature $T_{N}$ and superconducting electrode temperature $T_{S}$ plotted versus $T_{S} / T_{N}$ for $T_{N}=0.1$, 0.2 , and $0.3 \mathrm{~K}$. The result is normalized by $P_{N}$ evaluated with $T_{S}=T_{N}$. The bias is $0.95 \Delta / e$.

Previous experimental work described deviations from the expected thermal behavior in terms of a power load on the normal electrode $\beta P_{S}\left(T_{N}, T_{S}=T_{b}\right)$. [3] This description implies that a fraction $\beta$ of the power deposited in the superconductor, $P_{S}$, returns to the normal electrode. The fraction $\beta$ was measured to be $0.125-0.15$ for $T_{b}$ of $0.2-0.3 \mathrm{~K}$ and $V_{b}$ of $0-1.1 \Delta / e$. The same $\beta$ was found for 2 junctions with lengths of 20 and $15 \mu \mathrm{m}$, areas of 400 and $150 \mu \mathrm{m}^{2}$, and specific resistances of $3300 \Omega \mu \mathrm{m}^{2}$ and $7200 \Omega \mu \mathrm{m}^{2}$, respectively. We next describe how to calculate $\beta$.

To estimate the power load due to quasiparticle recombination and the loss in cooling power due to values of $T_{S}>T_{b}$, we calculate the quasiparticle density in the superconductor. This is equivalent to calculating $T_{S}$. The spatially varying excess quasiparticle density, $n_{x}(x)$, is given by $D \nabla^{2} n_{x}-\Gamma_{R}\left(n_{x}^{2}+2 n_{x} n_{t h}\right)+\Gamma_{Q P} g(x)=0$ where $D$ is the diffusion constant. The end of the junction is a mirror and the point where the superconductor overlaps a normal contact pad is a sink. The function $g(x)$ describes the injection geometry. The rate of quasiparticle injection, $\Gamma_{Q P}$, is given by $\mathrm{Eq}$. (1) without the factor of $E$ inside the integral. Since $\Gamma Q P$ is a function of $T_{S}$ and thus $n_{x}$, we solve for $n_{x}$ iteratively until a self-consistent solution is found.

Calculated values of $n_{x}$ due to currents of $1.67 \mu \mathrm{A}$ (solid line) and $6.84 \mu \mathrm{A}$ (dashed line) are plotted in Fig. 2. These currents correspond to $V_{b}=$ $0.75 \Delta / e$ and $1.0 \Delta / e$, and $T_{N}=0.3022$ and 0.3086 $\mathrm{K}$, respectively. The bath temperature was $0.304 \mathrm{~K}$. The injection region extends from $x=0$ to $x=20 \mu \mathrm{m}$ and the quasiparticle sink occurs at $x=47.5 \mu \mathrm{m}$. Including phonon trapping, we calculate $\Gamma_{R}=30$ $\mu \mathrm{m}^{3} / \mathrm{s}$. The diffusion constant $D$ is calculated from resistivity measurements and the quasiparticle dispersion relation. A value for $D$ of $9 \times 10^{9} \mu \mathrm{m}^{2} / \mathrm{s}$ is typical, but there is a weak dependence on $V_{b}, T_{N}$, and $T_{S}$. To simplify, we set $T_{S}$ equal to $T_{b}$ when calculating $D$. The excess densities of $10,500 \mu \mathrm{m}^{-3}$ and $37,300 \mu \mathrm{m}^{-3}$ in the junction correspond to $T_{S}=$ 0.371 and $0.447 \mathrm{~K}$, respectively.

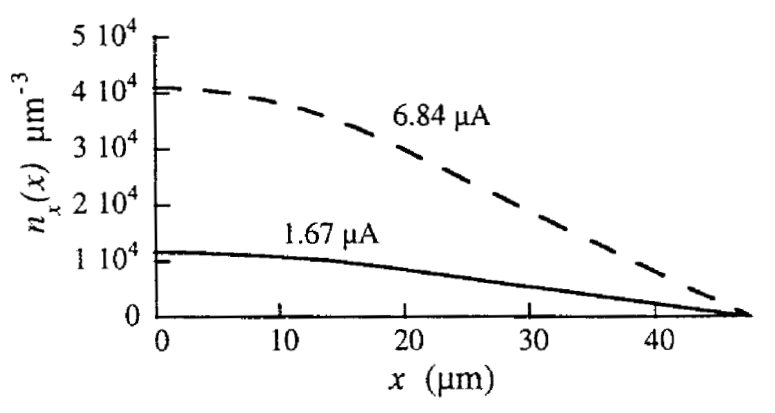

fig. 2 Excess quasiparticle density $n_{x}(x)$.

Given $n_{x}$ and $T_{S}$ in the superconducting electrode of the junction, we can calculate $\beta$. Based on phonon-electron and phonon escape rates, we estimate $p_{p-e}$ is 0.4 . We conclude that for $l=1.67$ $\mu \mathrm{A}$, recombination produces a power load of $4 \mathrm{pW}$ on the normal electrode. The decrease in cooling power due to elevation of $T_{S}$ above $T_{b}$ is also significant. We calculate from Eq. (1) that the cooling power is reduced by $11 \mathrm{pW}$. Summing these powers and dividing by Eq. (1) evaluated with $T_{S}=T_{b}$, we arrive at $\beta=.05$. For $I=6.84 \mu \mathrm{A}$, the recombination load is $37 \mathrm{pW}$, the decrease in cooling power is $42 \mathrm{pW}$, and $\beta$ is .06. These predictions for $\beta$ incorporate no free parameters and agree with measurement to factors of 2-3. The predictions are largely independent of bias, in good agreement with measurement. Calculations carried out at $T_{b}=0.226 \mathrm{~K}$ yield similar values of $\beta$. Hence, predictions for $\beta$ are independent of $T_{b}$ over the range $0.2-0.3 \mathrm{~K}$, also in good agreement with 
measurement. The predicted values of $\beta$ can be increased to $0.125-0.15$ by decreasing the mean free path by a factor near 3 .

The predicted values of $\beta$ for the $15 \mu \mathrm{m}$ long junction in [3] are smaller: .02-.03. It is surprising that $\beta$ measured in the $15 \mu \mathrm{m}$ and $20 \mu \mathrm{m}$ junctions is the same because of the higher specific resistance and shorter distance quasiparticles need diffuse before leaving the smaller junction. It is possible that the mean free path in the $\mathrm{Al}$ of the smaller junction is shorter than in the larger because shadow mask deposition produces more disorder in small features.

In conclusion, we have successfully developed techniques to calculate the degradation in NIS refrigerator performance caused by heating of the superconducting electrode. This heating can be reduced by using a thick, clean film for the electrode, by placing a quasiparticle trap near the junction, and by using a short junction.

\section{II-CRYOGEN-FREE REFRIGERATION}

Detectors operating below $1 \mathrm{~K}$ will be powerful tools for materials microanalysis because of their ability to accurately measure X-ray energies. [4] Cryogenic detectors may also prove useful for mass spectrometry of heavy biological molecules. [5] Both these activities are commonly pursued outside low temperature physics laboratories. As a result, there is a clear need for simple, reliable, closed-cycle refrigeration equipment. A candidate system might consist of a two stage adiabatic demagnetization refrigerator (ADR) capable of cooling from $4 \mathrm{~K}$ to $100 \mathrm{mK}$ coupled to a mechanical cooler capable of cooling from $300 \mathrm{~K}$ to $4 \mathrm{~K}$. While mechanical coolers able to reach $4 \mathrm{~K}$ have existed for a number of years, there has been persistent concern that the vibration produced by these coolers is incompatible with the operation of sensitive electronics. However, the recent development of pulse tube mechanical coolers capable of reaching $4 \mathrm{~K}$ has renewed interest in closed-cycle systems because of the low vibration of the pulse tube. [6] We have coupled a pulse tube to an ADR cooled by liquid cryogens in order to measure how the vibration of the pulse tube affects the resolution of superconducting tunnel junction (STJ) detectors.

We mounted a Cryomech PT4-05 pulse tube in a dewar adjacent to a second dewar containing a single stage ADR. A rigid cold finger joined the second stage of the pulse tube to the $4.2 \mathrm{~K}$ stage of the ADR. We measured $\mathrm{X}$-rays with $\mathrm{Nb}-\mathrm{Al}-\mathrm{AlOx}$ $\mathrm{Al}-\mathrm{Nb} \mathrm{STJ}$ both with and without the pulse tube operating at its base temperature of $2.8 \mathrm{~K}$. As shown in Fig. 3, the energy resolution of the STJs was degraded from $26 \mathrm{eV}$ to $30 \mathrm{eV}$ by the vibration of the pulse tube when measuring $1.04 \mathrm{keV} \mathrm{Na} \mathrm{K}$ shell Xrays. This degradation corresponds to a $15 \mathrm{eV}$ noise source added in quadrature. Spectral analysis of the detector baseline showed that operation of the pulse tube added several noise peaks between 3 and 50 $\mathrm{kHz}$. Since no effort was made to mechanically or electrically isolate the ADR from the pulse tube, we believe this first effort is encouraging. It is hoped these results will spur development of closed-cycle refrigeration systems for cryogenic detectors.

This work was performed under the auspices of the U.S. Department of Energy by Lawrence Livermore National Laboratory under contract No. W-7405-ENG-48.

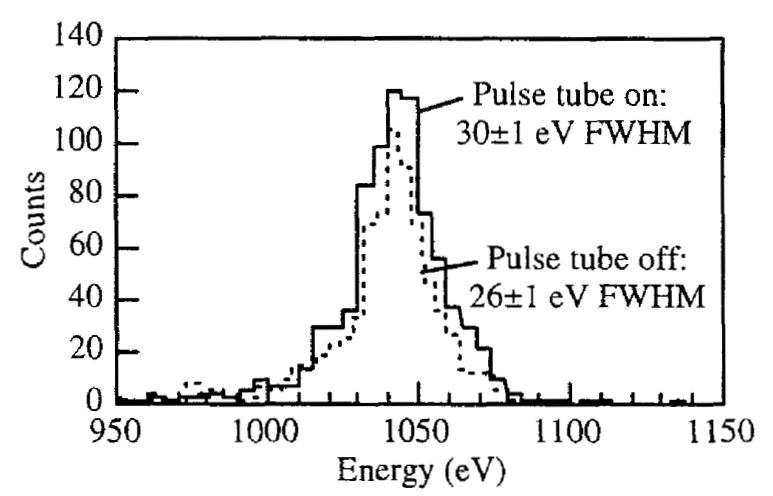

fig. 3 Effect of pulse tube vibrations on energy resolution of STJ detector for $1.04 \mathrm{keVX}$-rays.

\section{REFERENCES}

[1] M. Nahum, T. Eiles, and J.M. Martinis, Appl. Phys. Lett. 65 (1994) 3123.

[2] M.M. Leivo, J.P. Pekola, and D.V. Averin, Appl. Phys. Lett. 68 (1996) 1996, and subsequent papers by the same authors.

[3] P.A. Fisher, J.N. Ullom, and M. Nahum, Appl. Phys. Lett. 74 (1999) 2705.

[4] D.A. Wollman, K.D. Irwin, G.C. Hilton, L.L. Dulcie, D.E. Newbury, J.M. Martinis, J. of Microscopy 188 (1997) 196.

[5] D. Twerenbold, Nucl. Instr. and Meth. A 370 (1996) 253.

[6] Y. Matsubara, J.L. Gao, Cryogenics 34 (1994) 259. 